\title{
DESAFIOS ÉTICOS, METODOLÓGICOS E PRÁTICOS NA PESQUISA QUALITATIVA EM SAÚDE: UM OLHAR A PARTIR DE UM ESTUDO EM TORNO DO PROCESSO DE MORRER
}

\author{
ETHICAL, METHODOLOGICAL AND PRACTICAL CHALLENGES IN \\ QUALITATIVE HEALTH RESEARCH: A LOOK THROUGH A STUDY \\ AROUND THE DYING PROCESS
}

Ana Patrícia Hilário ${ }^{1}$

Fábio Rafael Augusto ${ }^{2}$

\begin{abstract}
Resumo: Partindo de uma experiência de investigação em torno do processo de morrer, este artigo procura discutir alguns dos desafios que emergiram no âmbito de uma pesquisa qualitativa de natureza etnográfica no campo da saúde. A sensibilidade do tema e a vulnerabilidade dos participantes conduziu à necessidade de criar estratégias que mitigassem potenciais efeitos indesejados para os atores envolvidos. Desta forma, discutem-se questões ético-metodológicas e o impacto que a pesquisa assumiu para os participantes e investigador. As principais conclusões apontam, por um lado, para a necessidade de criar diretrizes bem definidas no que diz respeito à pesquisa com sujeitos humanos e, por outro, para a importância de adequar os instrumentos de recolha no sentido de criar proximidade e salvaguardar o bemestar de todos os intervenientes. Este artigo procura contribuir para um debate em torno dos "bastidores" da pesquisa, dando a conhecer dilemas transversais à investigação.
\end{abstract}

Palavras-chave: Pesquisa qualitativa; Sensibilidade; Vulnerabilidade; Ética.

\begin{abstract}
Drawing upon a research on the dying process, this article seeks to discuss some of the challenges that have emerged in the context of a qualitative health research ethnography. The sensitivity of the topic and the vulnerability of the participants led to the need to create strategies to mitigate the potential undesirable effects for those involved in the research. The ethical and methodological aspects, as well as the impact that the research can assume for the participants and for the researcher are discussed. The conclusions point, on the one hand, to the need to create well-defined guidelines for research with human subjects and, on the other, to the importance of adapting the data collection instruments to create proximity and safeguard the well-being of all the actors. This article aims to contribute to the debate around the "backstage" of the research, revealing the dilemmas that across several studies.
\end{abstract}

Keywords: Qualitative research; Sensitivity; Vulnerability; Ethics.

\section{Introdução}

Este artigo tem por base uma investigação que procurou perceber como a deterioração corporal experienciada no período imediatamente antes da morte influencia

\footnotetext{
${ }^{1}$ Doutora em Sociologia da Saúde pela Royal Holloway, University of London (RHUL). Investigadora Auxiliar no Instituto de Ciências Sociais, Universidade de Lisboa (ICS-ULisboa), Lisboa, Portugal. patriciahilario@gmail.com

${ }^{2}$ Mestre em Sociologia: Exclusões e Políticas Sociais pela Universidade da Beira Interior (UBI). Doutorando em Sociologia no Instituto de Ciências Sociais, Universidade de Lisboa (ICS-ULisboa), Lisboa, Portugal. fabio.augusto@ics.ulisboa.pt
} 
o self e a identidade dos pacientes em fase terminal. Pretende-se aqui discutir alguns dos desafios inerentes ao desenvolvimento de uma pesquisa qualitativa de natureza etnográfica no campo da saúde com sujeitos que podem ser considerados vulneráveis e acerca de um tema que se pode pautar pela sensibilidade. A pesquisa qualitativa de natureza etnográfica tem, de facto, vindo a ser descrita como o método privilegiado para desenvolver estudos, quer com sujeitos vulneráveis, como é o caso dos pacientes em fase terminal (LAWTON, 2001), quer acerca de temas considerados sensíveis, como é o caso da doença (WATTS, 2008) e da morte (WOODTHORPE, 2009). Esta adaptabilidade do método advém, sobretudo, do seu carácter não invasivo, que permite ter em conta a temporalidade dos participantes do estudo, respeitando simultaneamente a emocionalidade inerente ao contexto e às situações vividas/experienciadas pelos próprios (LAWTON, 2001). Neste sentido, é importante compreender qual a melhor forma de desenvolver pesquisa com sujeitos vulneráveis e sobre temas sensíveis, salvaguardando tanto os participantes, como os próprios investigadores (ADDINGTON-HALL, 2002; BERRY, 2004). Assim sendo, neste artigo, num primeiro momento, explora-se o conceito de vulnerabilidade associado aos sujeitos ou grupos investigados e o conceito de sensibilidade associado ao tema ou tópicos de pesquisa abordados. Num segundo momento, enumeram-se as estratégias metodológicas e os princípios éticos que nortearam a investigação. Num terceiro momento, explora-se o impacto que a pesquisa teve para os vários intervenientes, discutindo-se algumas estratégias que podem contribuir para mitigar alguns efeitos indesejados.

\section{Vulnerabilidade e sensibilidade na pesquisa em torno do processo de morrer}

Levar a cabo uma investigação envolve dar resposta a uma multiplicidade de desafios, sejam eles de ordem metodológica, teórica, ética, política ou legal (LEE; RENZETTI, 1990). A forma como eles são ultrapassados depende, essencialmente, do contexto em que a pesquisa se insere e dos seus atores. Nesta "equação", o papel que os investigadores assumem pode (e tende) a ser decisivo, cabendo-lhes a responsabilidade de construir um projeto com valor científico e, simultaneamente, com valor social que vá ao encontro de princípios e direitos humanos considerados fundamentais (RAPOSO, 2020). Tais princípios e direitos orientadores referem-se, de modo geral, ao respeito pela pessoa (i.e., deve prevalecer a vontade individual, devendo ser dada especial atenção a quem se encontra numa situação de vulnerabilidade e/ou possui a sua capacidade de 
decisão diminuída), à beneficência/não maleficência (i.e., dever de não fazer mal, maximizando os benefícios e minimizando os riscos) e à justiça (i.e., não serem impostos fardos desnecessários, nem ser recusado qualquer benefício) (ALMEIDA, 2013). Para além disso, desenvolver investigação em torno do processo de morrer e, especificamente, acerca da relação que se estabelece entre a deterioração corporal experienciada no período imediatamente antes da morte e a (re)construção do self e a identidade dos pacientes em fase terminal, implica refletir acerca da (potencial) vulnerabilidade dos participantes envolvidos e da (eventual) sensibilidade do tema. Tal reflexão permite salvaguardar o bem-estar de todos os intervenientes e, por conseguinte, evitar danos a nível físico, psicológico e emocional.

De facto, é possível enquadrar os pacientes em fase terminal na categoria de grupo vulnerável (ADDINGTON-HALL, 2002), fundamentalmente, pela sua condição física debilitante e deteriorante que pode condicionar a autonomia e a capacidade para tomar certas decisões (MOORE; MILLER, 1999; SILVA, 1995). Apesar desta (potencial) vulnerabilidade, os pacientes em fase terminal podem, caso pretendam e caso a sua condição não coloque em risco o funcionamento cognitivo, participar em pesquisas no âmbito das ciências sociais, particularmente, sobre assuntos que lhes são próximos (ADDINGTON-HALL, 2002; BERRY, 2004; BLOOMER et al., 2017). Assim, é possível potencializar a fala dos participantes e pensar em estratégias que vão ao encontro das suas reais necessidades (DICKSON-SWIFT; JAMES; LIAMPUTTONG, 2008; LEE; RENZETTI, 1990). Ainda que os resultados da investigação e os benefícios que daí advêm possam não reverter diretamente para estes participantes, a possibilidade de ajudar outras pessoas que, direta ou indiretamente, partilham a mesma realidade (ou próxima) e o efeito catártico e/ou terapêutico que pode advir do diálogo entre investigadorinvestigado representam motivações válidas (ALEXANDER, 2010; AUGUSTO; HILÁRIO, 2019; BLOOMER et al., 2017). Desta forma, considera-se importante evitar a adoção de uma postura paternalista (desconsiderando a vontade do participante por intermédio da sua exclusão do processo de tomada de decisão) e olhar para o carácter contextual do conceito de vulnerabilidade - varia consoante a situação, o sujeito investigado, o investigador e a relação estabelecida entre eles -, onde se pressupõe que a vulnerabilidade assume diferentes formas e não representa, necessariamente, um elemento limitador/condicionador da pesquisa (BRACKEN-ROCHE et al., 2017; VON BENZON; VAN BLERK, 2017). Considera-se, assim, que os pacientes em fase terminal, em particular, os que foram investigados no âmbito da pesquisa em causa, constituem 
agentes ativos, críticos e reflexivos com a capacidade para tomar decisões acerca do papel que pretendem assumir e desempenhar na pesquisa e sobre os aspetos que querem abordar (BERRY, 2004).

Além da (potencial) vulnerabilidade dos participantes, destaca-se também a (eventual) sensibilidade do tema ou dos assuntos tratados no âmbito da pesquisa. Abordar o processo de morrer e, em particular, as experiências de deterioração corporal decorrentes desse mesmo processo pode suscitar algumas inquietações. Por se tratar de um tema que é, frequentemente, associado a sentimentos e emoções negativas - de perda, tristeza e saudade - e por incidir sobre experiências íntimas e, portanto, do foro privado; o processo de morrer pode ser enquadrado na categoria de tema sensível (LEE; RENZETTI, 1990; WELLINGS; BRANIGAN; MITCHELL, 2000). Também a possibilidade de acarretar certas consequências ou implicações para os participantes da pesquisa, como por exemplo, desconforto, permitem classificar o processo de morrer como sendo um tema sensível (LEE, 1993; LEE; RAYMOND, 2012; WELLINGS; BRANIGAN; MITCHELL, 2000). Contudo, se, por um lado, parecem existir certas condições que permitem incluir determinados tópicos na categoria de temas sensíveis, por outro, e à semelhança do conceito de vulnerabilidade, trata-se de um conceito contextual, na medida em que temas aparentemente inócuos podem tornar-se sensíveis e vice-versa; ou seja, a sensibilidade do tema varia consoante a situação e os atores envolvidos, onde, por exemplo, a forma como as questões são formuladas e dirigidas e a forma como elas são recebidas podem ter um papel mais decisivo, no que toca ao "trazer" sensibilidade para o tema, do que, propriamente, o teor do mesmo. Assim, ainda que o processo de morrer possa, à partida, no imaginário social promover sensibilidade junto dos atores da pesquisa, ela não representa, por si só, um elemento gerador de sensibilidade, dependendo de uma multiplicidade de fatores e (pre)disposições.

De modo geral, estes conceitos aqui discutidos diferem dos conceitos de vulnerabilidade e sensibilidade utilizados em contexto de saúde. No caso do primeiro conceito, este tende a ser usado, nesse contexto, de forma a descrever situações de risco, associado a susceptibilidades que os indivíduos podem ter em relação a sofrer danos na sua saúde (BERTOLOZZI et al., 2009). Por sua vez, o segundo conceito refere-se a uma medida probabilística de diagnosticar corretamente um caso clínico (CASTIEL, 2004). Não obstante o carácter contextual dos conceitos, partir para a realização de uma pesquisa acerca de um tema (eventualmente) sensível e/ou que incide sobre um grupo (potencialmente) vulnerável implica pensar em estratégias que procurem 
combater/minimizar os riscos associados a este tipo de investigação (DICKSON-SWIFT et al., 2008; VINCETT, 2018). A este respeito, destaca-se a partilha de experiências de pesquisa que permitam dar a conhecer os processos inerentes à mesma e a forma como os desafios encontrados foram ultrapassados/geridos (AUGUSTO; HILÁRIO, 2019; VINCETT, 2018). Assim, procura-se, no presente artigo, dar a conhecer os "bastidores" de uma pesquisa desenvolvida em torno do processo de morrer, bem como a forma como certos obstáculos ético-metodológicos foram ultrapassados.

\section{Um olhar sobre os aspetos éticos e metodológicos da pesquisa}

Nesta secção procurar-se-á, num primeiro momento, dar conta dos desafios éticos da pesquisa relacionados, por um lado, a uma ética processual e, por outro, a uma ética na prática. Num segundo momento, irá se discutir sobre o consentimento informado tanto ao nível formal, como informal.

\subsection{A ética processual e a ética na prática: o exemplo da observação participante}

A pesquisa qualitativa contempla, naturalmente, diversas questões éticas. No âmbito das quais é possível identificar aspetos ligados a uma ética processual e outros que se relacionam com uma ética na prática. A primeira dimensão remete para o conjunto de procedimentos formais necessários para entrar no campo de pesquisa e realizar a investigação, sendo que tais procedimentos geralmente envolvem o contato com uma comissão de ética de forma a obter a sua apreciação. A segunda dimensão relaciona-se com as obrigações éticas que o investigador tem para com os participantes, bem como todo um conjunto de questões ou dilemas que surgem no decurso das interações quotidianas que são estabelecidas no campo de pesquisa, podendo estas emergir de forma totalmente inesperada. No seio desta imprevisibilidade, podem surgir "momentos eticamente relevantes/importantes", ou seja, situações marcadas pela inexistência de uma resposta óbvia e clara, mas com sérias implicações éticas (GUILLEMIN; GILLAM, 2004). Como seria de esperar, estas dimensões relacionam-se entre si e nem sempre é possível separá-las de forma clara. Sabendo-se que a investigação não se pauta apenas pelo formalismo burocrático e previsibilidade, cabe ao investigador desenvolver de forma crítica e reflexiva as suas ferramentas e estratégias de atuação em momentos nos quais as questões éticas se complexificam e a clareza se dissipa (ELLIS, 2010; HOLLAND; WILLIAMS; FORESTER, 2014; RORIZ; PADEZ, 2017; ROSSMAN; RALLIS, 2010). 
A investigação aqui descrita parte de uma pesquisa etnográfica realizada durante 10 meses em duas unidades de cuidados paliativos localizadas em Lisboa. Uma vez que o trabalho de campo iria ter lugar em duas unidades de cuidados paliativos privadas a atuar em Portugal, não foi necessário elaborar um pedido de autorização junto do Ministério da Saúde Português, nem obter consentimento prévio de nenhuma comissão de ética nacional. No entanto, na medida em que o estudo estava a ser desenvolvido no âmbito de uma pesquisa na Royal Holloway, University of London, foi necessário pedir autorização ao comité de ética dessa instituição.

As considerações apresentadas pela comissão de ética ao nível da proteção dos participantes no estudo evidenciaram ter por base um regime de regulamentação antecipada e, por conseguinte, incidiram no modelo proposto pela investigação clínica e de experimentação biomédica (MURPHY; DINGWALL, 2007). Tal regime de regulação assenta no pressuposto de que os participantes no estudo são indivíduos isolados cujas ações são passíveis de ser controladas pelo investigador. Todavia, no método etnográfico as situações de pesquisa não incidem sobre um conjunto delimitado à priori de indivíduos, mas sobre a dinâmica de certas relações e processos. Assim sendo, não é possível identificar todos os atores e antecipar todas as interações e situações que terão lugar no decurso da observação (GUILLEMIN; GILLAM, 2004).

A imposição feita pela comissão de ética de obrigatoriedade de obtenção do consentimento prévio por escrito à observação revela um desconhecimento sobre a natureza do método etnográfico. A noção de "rapport", que se refere a um cultivar de relações de proximidade e confiança, sendo tal um dos principais pilares da pesquisa etnográfica (GAGLIO; NELSON; KING, 2006), foi fortemente constrangida dada a imposição de consentimento prévio por escrito (NUNES, 2011). Este pedido comprometeu a quantidade e a qualidade da informação recolhida através da técnica de observação participante, na medida em que somente foi possível fazer o registo de certas situações (i.e., reuniões de equipa, interações entre equipa e pacientes; interações entre pacientes e familiares; interações entre a tríade: equipa, familiares, pacientes) com sujeitos que já haviam dado o seu consentimento prévio por escrito. No entanto, de modo a criar uma relação de proximidade e confiança, foi obtido o consentimento por escrito para a realização da observação após a condução das entrevistas aos sujeitos. A obtenção de consentimento prévio por escrito à observação no âmbito da pesquisa etnográfica não revelou ser um procedimento adequado (RAPOSO, 2016), indo ao encontro do que já havia sido assinalado na investigação em ciências sociais (MURPHY; DINGWALL, 
2007). Além disso, e de acordo com as diretrizes lançadas, em 2015, pela Comissão Europeia num documento intitulado "Research Ethics in Ethnography/Anthropology", denota-se a possibilidade de incorporar no âmbito de uma investigação formas "alternativas" de obtenção do consentimento dos participantes, destacando-se, por exemplo, o consentimento verbal. Este tipo de consentimento preserva o direito do participante de escolher participar e, simultaneamente, permite cultivar uma relação de confiança e de maior proximidade pela forma natural como é obtido (IPHOFEN, 2015).

No âmbito da prática etnográfica, o consentimento é visto como negociado e processual (RORIZ; PADEZ, 2017). Na verdade, deve-se encarar o consentimento como um processo que ocorre ao longo de toda a pesquisa (ELLIS, 2007), o que permitirá que os participantes tenham um maior controlo sobre a sua participação no estudo e sobre as decisões que tomam no âmbito do mesmo (WARIN, 2011). Embora pudesse ter sido benéfico para a investigação observar todos os pacientes e familiares ao longo de todo o processo de deterioração corporal e no momento específico da morte, tal não foi possível devido a limitações de tempo e de financiamento. Além disso, os familiares com os quais o investigador teve um contato mais prolongado e sobre os quais incidiu a maioria das observações são aqueles cujo familiar se encontrava numa situação mais crítica, sendo nesta fase que as famílias tendem a estar mais presentes e a prestar mais apoio.

\subsection{A formalidade versus a informalidade do consentimento informado: o caso das entrevistas}

À técnica de observação participante associou-se a condução de entrevistas semiestruturadas a 10 pacientes oncológicos em fase terminal, 20 familiares e 20 membros da equipa de cuidados paliativos (pertencentes a diversas categorias profissionais). Um dos requisitos imposto pela comissão de ética dizia respeito à obtenção de consentimento informado para a realização das entrevistas. De forma a obtê-lo, foi disponibilizado um folheto informativo aos participantes onde constavam os objetivos, métodos, riscos e benefícios do estudo. Deste modo, era possível garantir que a decisão de participar na investigação ocorria de forma consciente e informada (RICHARDS; SCHWARTZ, 2002; SEYMOUR; SKILBECK, 2002). Toda a informação disponibilizada em formato físico era também apresentada verbalmente, de modo a assegurar que os sujeitos a compreendiam (FRAVEL; BOSS, 1992). 
O pedido de consentimento por escrito foi encarado com estranheza por parte tanto de pacientes como de familiares. À exceção dos profissionais de saúde que evidenciaram estar, de certo modo, familiarizados com o processo na medida em que é utilizado frequentemente na pesquisa clínica e na investigação biomédica com humanos. A estranheza manifestada poderá estar relacionada com a natureza da sociedade portuguesa. De modo geral, a assinatura de um contrato remete para um ato de elevada seriedade em que a quebra de direitos e deveres acarreta, geralmente, consequências graves para ambas as partes envolvidas (SÃO JOSÉ; TEIXEIRA, 2013). Esta estranheza inicial esvaneceuse após se ter explicado aos participantes a importância e o papel do consentimento por escrito.

O consentimento foi obtido sob o entendimento de que a participação no estudo pelos sujeitos era voluntária (CASSARETT; KARLAWISH, 2000) e que poderiam desistir da sua participação a qualquer momento sem prejuízo (TEE; LATHLEAN, 2004). Foi assegurado que os sujeitos se sentiam confortáveis com o facto de as entrevistas serem gravadas e, posteriormente, transcritas. À semelhança do que tem sido descrito como uma boa prática na literatura em torno do processo de morrer, foi dada aos pacientes a possibilidade de usarem os seus próprios nomes de forma a imortalizar a sua história (HOCKEY, 2007). Todavia, esta não foi uma opção considerada pelos participantes, que preferiram manter-se anónimos. Isto pode, eventualmente, ser explicado pelo desconhecimento que os pacientes entrevistados tinham sobre a iminência da sua morte, sendo que a maior parte deles não foram informados sobre o prognóstico da sua doença.

Destaca-se a importância do diálogo informal estabelecido com os profissionais de saúde que permitiu a obtenção de informações sobre o estado clínico dos pacientes e sobre o conhecimento que estes possuíam acerca da sua condição, possibilitando gerir as emoções dos participantes e evitar a transmissão de informações (potencialmente) nocivas. No decorrer da entrevista, optou-se por não se usar palavras como "deterioração corporal", "doença terminal", "processo de morrer" e "morte" pois tal poderia dar indicação sobre a gravidade da situação e iminência da morte (KENDALL et al., 2007). Contudo, um dos pacientes indagou o investigador no final da entrevista acerca do prognóstico da sua doença. Dado que somente os profissionais de saúde podem dar informações específicas sobre o estado de saúde, o investigador solicitou que o paciente remetesse tal questão para a equipa clínica. O paciente interrogou, de facto, a equipa clínica e foi lhe transmitido o seu prognóstico, o que lhe permitiu preparar-se para a sua morte iminente, em particular que comunicasse as suas últimas vontades e desejos aos 
seus entes queridos. Destaca-se, assim, como o carácter reflexivo da entrevista pode conduzir à modificação de atitudes e comportamentos por parte dos entrevistados.

\section{0 impacto da pesquisa nos seus atores: estratégias de mitigação}

Nesta secção, pretende-se refletir sobre o impacto que a pesquisa teve quer nos participantes, quer no investigador. Assim, num primeiro momento, descreve-se as estratégias adotadas de modo a minimizar o impacto negativo que a investigação pudesse ter sobre os participantes. Por sua vez, num segundo momento, apresenta-se as estratégias desenvolvidas pelo investigador de forma a salvaguardar também o seu bem-estar.

\subsection{O impacto da pesquisa nos participantes}

Dada a natureza (eventualmente) sensível das questões abordadas, foram tomadas medidas com o propósito de proteger os participantes do estudo de qualquer impacto negativo que a investigação pudesse ter sobre os mesmos. De forma a minimizar o desenvolvimento de sentimentos negativos por parte dos sujeitos durante a recolha de dados procurou-se estabelecer uma relação de proximidade e confiança com eles. A noção de empatia assume aqui um papel central, sendo descrita como o colocar-se no lugar do outro, podendo ser expressa tanto a nível verbal como não-verbal (HAKANSSON; MONTGOMERY, 2003). Através do toque, sempre que ele se mostrou apropriado face à situação e à receptividade do participante, foi possível diminuir sentimentos negativos quando foram abordadas questões (potencialmente) sensíveis e dolorosas para os sujeitos. Escutar com preocupação e compaixão, bem como a manutenção do contato através do olhar foram outras estratégias empáticas adotadas (WATTS, 2008). Este clima de confiança e conforto que se traduziu no desenvolvimento de uma relação empática entre investigador e participantes foi fundamental para a concretização das entrevistas, e em particular para a obtenção de informação de qualidade (FERREIRA, 2014; PEGADO; ZÓZIMO; LOPES, 2016).

A reação mais comum dos participantes, nomeadamente dos pacientes entrevistados, foi de incerteza para com a doença. A procura de validação de que tudo acabaria por ficar bem dominou as narrativas dos pacientes, bem como as dos seus familiares (WATTS, 2008). A grande maioria dos sujeitos indicara que a participação no estudo foi benéfica, uma vez que permitiu que discutissem abertamente sobre as suas experiências do cuidado em fim de vida e as emoções associadas ao processo de morrer 
(DICKSON-SWIFT et al., 2006; RAGER, 2005). Tal situação foi, particularmente, evidente ao nível das entrevistas tendo sido realçado por membros da equipa de cuidados paliativos, nomeadamente por enfermeiros e psicólogos, o efeito catártico e/ou terapêutico que as mesmas tiveram sobre si mesmos e sobre os vários participantes no estudo (DICKSON-SWIFT et al., 2008).

As entrevistas ao nível da investigação criam, de certo modo, um ambiente semelhante às entrevistas terapêuticas, na medida em que providenciam um espaço onde os indivíduos comunicam de forma espontânea sem receio de crítica ou julgamento por parte de quem escuta (DICKSON-SWIFT et al., 2006; LOWES; GILL, 2006). A procura de uma linguagem acessível, que fosse ao encontro do conhecimento que os entrevistados tinham sobre a sua situação ou sobre a situação dos seus familiares, foi uma preocupação constante ao longo de toda a pesquisa.

A grande maioria dos entrevistados mencionara que a sua participação no estudo se prendia sobretudo a motivos altruístas, uma vez que apesar dele não os beneficiar diretamente, confiavam que o estudo iria melhorar os cuidados prestados no futuro a pacientes e/ou a familiares que se encontrassem em situações semelhantes (LOWES; GILL, 2006). Denotou-se, portanto, a importância de transmitir o valor da pesquisa para os participantes.

\section{2 $O$ impacto da pesquisa no investigador}

O desenvolvimento de pesquisa em torno de tópicos considerados sensíveis como o processo de morrer pode ter um impacto profundo no bem-estar dos investigadores (BAHN; WEATHERILL, 2012; DICKSON-SWIFT et al., 2008; LALOR; BEGLEY; DEVANE, 2006). A proximidade com a morte e a escuta das narrativas de pacientes e familiares coloca os investigadores num campo repleto de emoções e exige da sua parte uma grande destreza emocional (VISSER, 2017). Não só os investigadores têm de lidar com os sentimentos dos entrevistados, mas também com os seus próprios sentimentos (WATTS, 2008; WOODTHORPE, 2009). Tal remete para a noção de "trabalho emocional" desenvolvida por Hochschild (1983) que, de modo geral, se refere ao esforço consciente ou não, neste caso desenvolvido pelos investigadores, no sentido de gerir as suas emoções e sentimentos.

Na verdade, o contato prolongado com a temática da morte pode gerar medos nos investigadores acerca da sua própria morte ou da morte de familiares e amigos próximos 
(CLARKE; INGLETON; SEYMOUR, 2000; JOHNSON; CLARKE, 2003). Assim sendo, num primeiro momento da investigação, antes da entrada no campo, os investigadores são levados a questionar-se sobre os seus sentimentos e reações perante a morte. Apesar da forte carga emocional associada a tais questões, este exercício, no âmbito da pesquisa, permitiu que se refletisse sobre como se iria responder face à proximidade com o fenómeno, preparando o investigador, de certo modo, para o inevitável confronto com o campo.

Já no campo, com o propósito de minimizar o impacto subjacente à proximidade com a morte, foi desenvolvido um conjunto de estratégias. Em primeiro lugar, salientase a elaboração de um diário de campo onde foram relatados os sentimentos e emoções experienciados ao longo dos 10 meses do trabalho de campo, tendo-se revelado uma ferramenta de reflexão de enorme importância (VALENTINE, 2007). O contato com outros colegas de investigação permitiu discutir acerca dos desafios encontrados no decorrer do estudo através da partilha de experiências (ROLLS; RELF, 2006). Não obstante, o processo de pesquisa revelou ser bastante recompensador, na medida em que permitiu que os pacientes, os familiares e os profissionais de saúde pudessem contar a sua história. Foram experienciados sentimentos de gratidão pela partilha, por parte dos participantes de momentos importantes, o que contribuiu para a validação do estudo desenvolvido (DICKSON-SWIFT et al., 2008).

\section{Considerações finais}

O presente artigo corrobora a ideia de que a ética não se deve traduzir em instrumentos marcadamente burocráticos (MONAGHAN; O’DWYER; GABE, 2013), que remetem para a necessidade intrínseca de uma homogeneização de procedimentos característicos do modelo biomédico (MINAYO; GUERRIERO, 2014). No decorrer da pesquisa, podem ocorrer situações com menor previsibilidade, que Guillemin e Gillam (2004) denominam de "momentos eticamente relevantes/importantes", pelo que o investigador deve então ter a liberdade para se socorrer da sua sensibilidade moral e emocional (ELLIS, 2010) para dar resposta a dilemas que se prendem de certa forma a essa "microética" (HOLLAND; WILLIAMS; FORESTER, 2014). A ética, que se traduz na reflexividade do próprio investigador, deve, pois, ser vista como ferramenta essencial em todo o processo de pesquisa (ROSSMAN; RALLIS, 2010). Esta reflexividade foi evidente desde a construção do desenho da pesquisa até ao decorrer do trabalho de campo, 
sobretudo de modo a minimizar o impacto negativo que uma investigação sobre temas sensíveis pode ter não só nos participantes, como no próprio investigador (EMERALD; CARPENTER, 2015). Neste sentido, foram desenvolvidas estratégias que contribuíram, de certo modo, para mitigar esse impacto. Salienta-se, por exemplo, o desenvolvimento de uma relação de proximidade e confiança entre os participantes e o investigador. A preparação do investigador para o campo permitiu também a minimização de sentimentos negativos que pudessem surgir no desenvolvimento da pesquisa etnográfica dado o tema e a população.

Foi através da reflexividade que o investigador conseguiu dar resposta quer às questões da ética processual, quer aos dilemas que advêm da ética na prática. Na verdade, a reflexividade permitiu ultrapassar as tensões éticas que surgiram no decorrer da investigação (RORIZ; PADEZ, 2017). Porém, não se pretende neste artigo ir contra a existência de uma ética processual, mas dar continuidade ao debate acerca da importância da ética na prática na pesquisa em ciências sociais. Procurou-se, assim, apresentar alguns dos desafios de ordem ética e metodológica que o investigador pode enfrentar neste tipo de pesquisa. O recurso à pesquisa etnográfica, em particular à observação participante e a entrevistas semi-estruturadas, confirmou-se como a opção metodológica mais adequada para desenvolver investigação sobre temas (eventualmente) sensíveis com populações (potencialmente) vulneráveis. As especificidades da aplicação do consentimento informado por escrito no âmbito da pesquisa etnográfica foram abordadas no presente artigo. Estas especifidades corroboram a importância de existirem diretrizes claras, mas suficientemente abertas que permitam ter em consideração o caracter contextual deste tipo de pesquisa.

Este artigo procurou dar visibilidade a questões que têm vindo a ser remetidas para segundo plano no âmbito dos debates em ciências sociais, onde se privilegia a apresentação e discussão dos resultados e não se atribui o devido enfâse aos dilemas associados ao processo de pesquisa. Realizar investigação sobre o modo como a deterioração corporal influência o self e a identidade dos doentes em fase terminal implica pensar quer sobre a sensibilidade do tema e a vulnerabilidade dos participantes, quer sobre os procedimentos éticos e metodológicos a adoptar neste tipo de pesquisa de modo a cumprir os objetivos de investigação propostos e respeitar o rigor científico. Pretendese, através deste artigo, abrir o mote para um debate alargado sobre estas questões, em particular com investigadores em início de carreira, que devido à sua menor experiência podem se encontrar menos preparados para lidar com os vários desafios éticos e 
metodológicos decorrentes de desenvolver investigação sobre um tema (eventualmente) sensível e com uma população (potencialmente) vulnerável.

\section{Referências}

ADDINGTON-HALL, J. Research sensitivities to palliative care patients. European Journal of Cancer Care, Oxford, v. 11, n. 3, p. 220-224, set. 2002.

ALEXANDER, S. 'As long as it helps somebody': why vulnerable people participate in research. International Journal of Palliative Nursing, London, v. 16, n. 4, p. 173-178, abr. 2010.

ALMEIDA, J. Ética da investigação em ciências sociais. Sociologia Online, Lisboa, n. 6, s/p, jun. 2013.

AUGUSTO, F. R.; HILÁRIO, A. P. 'Through the Looking Glass': The Emotional Journey of the Volunteer Ethnographer when Researching Sensitive Topics with Vulnerable Populations. The Qualitative Report, Flórida, v. 24, n. 13, p. 17-30, abr. 2019.

BAHN, S.; WEATHERILL, P. Qualitative social research: a risky business when it comes to collecting 'sensitive' data'. Qualitative Research, Cardiff, v. 13, n. 1, p. 9-35, ago. 2012.

BERRY, S. For purposes of research, palliative care patients should not be considered a vulnerable population. Clinical Oncology, Alexandria, v. 16, n. 3, p. 223-224, fev. 2004.

BERTOLOZZI, M. et al. Os conceitos de vulnerabilidade e adesão na Saúde Colectiva. Rev Esc Enfermagem USP, São Paulo, v. 43, n. spe2, p. 1326-1330, dez. 2009.

BLOOMER, M. et al. Dying persons' perspectives on, or experiences of, participating in research: An integrative review. Palliative Medicine, London, v. 32, n. 4, p. 851-860, dez. 2017.

BRACKEN-ROCHE, D. et al. The concept of 'vulnerability' in research ethics: an in-depth analysis of policies and guidelines. Health Research Policy and Systems, London, v. 15, n. 8, p. 1-18. fev. 2017.

CASSARETT, D.; KARLAWISH, J. Are special ethical guidelines needed for palliative care research? Journal of Pain and Symptom Management, Plymouth, v. 20, n. 2, p. 130-139. ago. 2000.

CASTIEL, L. Promoção de saúde e a sensibilidade epistemológica da categoria 'comunidade'. Rev Saúde Pública, São Paulo, v. 38, n. 5, p. 615-622, out. 2004.

CLARK, D.; INGLETON, C.; SEYMOUR, J. Support and supervision in palliative care research. Palliative Medicine, London, v. 14, n. 5, p. 441-446, jul. 2000.

DICKSON-SWIFT, V. et al. Blurring boundaries in qualitative health research on sensitive topics. Qualitative Health Research, Salt Lake City, v. 16, n. 6, p. 853-871, jul. 2006.

DICKSON-SWIFT, V. et al. Researching sensitive topics: qualitative research as emotion work. Qualitative Research, Cardiff, v. 9, n. 1, p. 61-79, fev. 2009. 
DICKSON-SWIFT, V. et al. Risk to Researchers in Qualitative Research on Sensitive Topics: Issues and Strategies. Qualitative Health Research, Salt Lake City, v. 18, n. 1, p. 133-144, jan. 2008.

DICKSON-SWIFT, V.; JAMES, E.; LIAMPUTTONG, P. Undertaking Sensitive Research in the Health and Social Sciences: Managing Boundaries, Emotions and Risks. Cambridge: Cambridge University Press, 2008.

ELLIS, C. Telling secrets, revealing lives: Relational ethics in research with intimate others. Qualitative Inquiry, London, v. 13, n. 1, p. 3-29, jan. 2007.

ELLIS, J. Family practices during life-threatening illness: exploring the everyday. 2016. 435f. Tese (Doutorado em Sociologia) - Faculdade de Ciências Sociais, Universidade de Sheffield, Sheffield, 2010.

EMERALD, E.; CARPENTER, L. Vulnerability and emotions in research: risk, dilemmas, and doubts. Qualitative Inquiry, London, v. 21, n. 8, p. 741-750, mar. 2015.

FERREIRA, V. Artes e manhas da entrevista compreensiva. Saúde e Sociedade, São Paulo, v. 23, n. 3, p. 979-992, jul/set. 2014.

FRAVEL, D.; BOSS, P. An in-depth interview with the parents of missing children. In: GILGUN, J.; DALY, K.; HANDEL, G. (ed.). Qualitative Methods in Family Research. Newbury Park, CA: Sage, 1992. p. 126-145.

GAGLIO, B.; NELSON, C.; KING, D. The role of rapport: lessons learned from conducting research in primary care setting. Qualitative Health Research, Salt Lake City, v. 16, n. 5, p. 723-734, maio. 2006.

GUILLEMIN, M.; GILLAM, L. Ethics, Reflexivity, and 'Ethically Important Moments' in Research. Qualitative Inquiry, London, v. 10, n. 2, p. 261-280, abr. 2004.

HAKANSSON, J.; MONTGOMERY, H. Empathy as an Interpersonal Phenomenon. Journal of Social and Personal Relationships, London, v. 20, n. 3, p. 267-284, jun. 2003.

HOCHSCHILD, A. The Managed Heart: Commercialization of Human Feeling. Berkeley, CA: University of California Press, 1983.

HOCKEY, J. Closing in on death? Reflections on research and researchers in the field of death and dying. Health Sociology Review, London, v. 16, n. 5, p. 436-446, jul. 2007.

HOLLAND, S.; WILLIAMS, A.; FORESTER, D. Navigating ethical moments when researching substance misuse with parents and their children. Qualitative Research, Cardiff, v. 14, n. 4, p. 411-427, ago. 2014.

IPHOFEN, R. Research ethics in ethnography/anthropology. Brussel: European Commission, DG Research and Innovation, 2015.

JOHNSON, B.; CLARKE, J. Collecting sensitive data: the impact on researchers. Qualitative Health Research, Salt Lake City, v. 13, n. 3, p. 421-434, mar. 2003.

KENDALL, M. et al. Key challenges and ways forward in researching the 'good death': qualitative in-depth interview and focus group. BMJ, London, v. 334, n. 521, p. 1-6, mar. 2007. 
LALOR, J.; BEGLEY, C.; DEVANE, D. Exploring painful experiences: impact of emotional narratives on members of a qualitative research team. Journal of Advanced Nursing, Oxford, v. 56, n. 6, p. 607-616, dez. 2006.

LAWTON, J. Gaining and maintaining consent: ethical concerns raised in a study of dying patients. Qualitative Health Research, Salt Lake City, v. 11, n. 5, p. 693-705, set. 2001.

LEE, R. Doing Research on Sensitive Topics. London: Sage Publications, 1993.

LEE, R.; RENZETTI, C. The Problems of Researching Sensitive Topics: An Overview and Introduction. American Behavioral Scientist, Thousand Oaks, v. 33, n. 5, p. 510-528, maio. 1990.

LEE, Y.; RAYMOND, L. Methodological Research on 'Sensitive' Topics: A Decade Review. Bulletin de Méthodologie Sociologique, London, v. 114, n. 1, p. 35-49, abr. 2012.

LOWES, L.; GILL, P. Participants' experiences of being interviewed about an emotive topic. Journal of Advanced Nursing, Oxford, v. 55, n. 5, p. 587-594, set. 2006.

MINAYO, M.; GUERRIERO, I. Reflexividade como éthos da pesquisa qualitativa. Ciência \& Saúde Coletiva, Rio de Janeiro, v. 19, n. 4, p. 1103-1112, abr. 2014.

MONAGHAN, L.; O’DWYER, M.; GABE, J. Seeking university Research Ethic Committee approval: the emotional vicissitudes of a 'rationalised' process. International Journal of Social Research Methodology, London, v. 16, n. 1, p. 65-80, jan. 2013.

MOORE, L.; MILLER, M. Initiating research with doubly vulnerable populations. Journal of Advanced Nursing, Oxford, v. 30, n. 5, p. 1034-1040, jan. 1999.

MURPHY, E.; DINGWALL, R. The Ethics of Ethnography. In: ATKINSON, P.; COFFEY, A.; DELAMONT, S.; LOFLAND, J.; LOFLAND, L. (ed.). Handbook of Ethnography. London: Sage, 2007. p. 339-351.

NUNES, J. Sobre a ética (e a política) da investigação social em saúde. Sociologia On Line, Lisboa, n. 3, p. 167-188, jun. 2011.

PEGADO, E.; ZÓZIMO, J.; LOPES, N. Histórias de (uma) vida: desafios e dilemas éticos na investigação com idosos. Sociologia On Line, Lisboa, n. 12, p. 5-21, dez. 2016.

RAGER, K. Self-care and the qualitative researcher: when collecting data can break your heart. Educational Researcher, Washington, v. 34, n. 4, p. 23-27, maio. 2005.

RAPOSO, H. Apreciação ética de projectos de investigação em saúde: uma reflexão situada a propósito da ética de investigação em ciências sociais. Sociologia On Line, Lisboa, n. 12, p. 22 48, dez. 2016.

RAPOSO, H. Tensões e desafios da eticidade da investigação científica em saúde: uma reflexão em aberto. Saúde e Tecnologia, Lisboa, n. 23, p. 19-26, maio. 2020.

RICHARDS, H.; SCHWARTZ, L. Ethics of qualitative research: are there special issues for health services research? Family Practice, Oxford, v. 19, n. 2, p. 135-139, abr. 2002.

ROLLS, L.; RELF, M. Bracketing interviews: addressing methodological challenges in qualitative interviewing in bereavement and palliative care. Mortality, London, v. 11, n. 3, p. 286-305, ago. 2006. 
RORIZ, M.; PADEZ, C. A regulação ética da investigação e os desafios postos às práticas etnográficas. Etnográfica, Lisboa, v. 21, n. 1, p. 75-95, mar. 2017.

ROSSMAN, G.; RALLIS, S. Everyday ethics: reflections on practice. International Journal of Qualitative Studies in Education, London, v. 23, n. 4, p. 379-391, maio. 2010.

SÃO JOSÉ, J.; TEIXEIRA, A. In the 'ethical crossroads' of ethnography: observing the care encounter at the ender's home. In: Paoletti, I.; Tomás, M.; Menéndez, F. (ed.). Practice of Ethics: an empirical approach to ethics in social science research. Newcastle: Cambridge Scholar Publishing, 2013. p. 43-63.

SEYMOUR, J.; SKILBECK, J. Ethical considerations in researching user views. European Journal of Cancer Care, Oxford, n. 11, p. 215-219, set. 2002.

SILVA, M. Ethical Guidelines in the Conduct, Dissemination and Implementation of Nursing Research. Washington, DC: American Nurses Publishing, 1995.

TEE, S.; LATHLEAN, J. The ethics of conducting a co-operative inquiry with vulnerable people. Journal of Advanced Nursing, Oxford, v. 47, n. 5, p. 536-543, set. 2004.

VALENTINE, C. Methodological reflections: attending and tending to the role of the researcher in the construction of bereavement narratives. Qualitative Social Work, Los Angeles, v. 6, n. 2, p. 159-176, jun. 2007.

VINCETT, J. Researcher self-care in organizational ethnography: Lessons from overcoming compassion fatigue. Journal of Organizational Ethnography, Bingley, v. 7, n. 1, p. 44-58, abr. 2018.

VISSER, R. 'Doing death': Reflecting on the researcher's subjectivity and emotions. Death Studies, London, v. 41, n. 1, p. 6-13, maio. 2017.

VON BENZON, N.; VAN BLERK, L. Research relationships and responsibilities: 'Doing' research with 'vulnerable' participants: introduction to the special edition. Social \& Cultural Geography, London, v. 18, n. 7, p. 895-905, jul. 2017.

WARIN, J. Ethical mindfulness and reflexivity: managing a research relationship with children and young people in a 14-year qualitative longitudinal research (QLR) study. Qualitative Inquiry, London, v. 17, n. 9, p. 805-814, out. 2011.

WATTS, J. Emotion, empathy and exit: reflections on doing ethnographic qualitative research on sensitive topics. Medical Sociology Online, Liverpool, v. 3, n. 2, p. 3-14. 2008.

WELLINGS, K.; BRANIGAN, P.; MITCHELL, K. Discomfort, Discord and Discontinuity as Data: Using Focus Groups to Research Sensitive Topics. Culture, Health \& Sexuality, London, v. 2, n. 3, p. 255-267, nov. 2000.

WOODTHORPE, K. Reflecting on death: The emotionality of the research encounter. Mortality, London, v. 14, n. 1, p. 70-86, fev. 2009.

Recebido em: 08 de novembro de 2020.

Aceito em: 28 de março de 2021. 